\title{
BANACH SPACES WITH SMALL CALKIN ALGEBRAS
}

\author{
MANUEL GONZÁLEZ \\ Departamento de Matemáticas, Universidad de Cantabria \\ E-39071 Santander, Spain \\ E-mail: gonzalem@unican.es
}

\begin{abstract}
Let $X$ be a Banach space. Let $\mathcal{A}(X)$ be a closed ideal in the algebra $\mathcal{L}(X)$ of the operators acting on $X$. We say that $\mathcal{L}(X) / \mathcal{A}(X)$ is a Calkin algebra whenever the Fredholm operators on $X$ coincide with the operators whose class in $\mathcal{L}(X) / \mathcal{A}(X)$ is invertible. Among other examples, we have the cases in which $\mathcal{A}(X)$ is the ideal of compact, strictly singular, strictly cosingular and inessential operators, and some other ideals introduced as perturbation classes in Fredholm theory.

Our aim is to present some classes of Banach spaces and some concrete examples of Banach spaces for which some of their Calkin algebras are "small" in some sense: finite dimensional, commutative, etc. The first example of such a Banach space was constructed around 1990. However, at this moment there is a great variety of examples of spaces of this kind, which provides interesting examples and counterexamples of operators. Moreover, the methods and techniques of operator theory have been found to be useful in the study of these spaces.
\end{abstract}

1. Introduction. In [GowersM:93], Gowers and Maurey presented an example of an infinite dimensional Banach space $X_{G M}$ (Example 6.1.1) such that no subspace of $X_{G M}$ can be written as the direct sum of two infinite dimensional closed subspaces. The existence of Banach spaces of this kind (which are called hereditarily indecomposable) was quite unexpected. It provided a negative answer to several long-standing open problems in Banach space theory. Moreover, for every operator $T$ acting on the complex version of $X_{G M}$ we can find a complex number $\lambda$ so that $T=\lambda I+S$, with $S$ a strictly singular operator. Afterwards, many examples of hereditarily indecomposable spaces and some other classes of spaces with unexpected properties were constructed. Now it is common

2000 Mathematics Subject Classification: Primary 47A53, 47L20; Secondary 46B03.

Key words and phrases: Calkin algebra, essential spectrum, semi-Fredholm operator, hereditarily indecomposable Banach space.

Research supported in part by DGI (Spain), Grant MTM2005-03831.

The paper is in final form and no version of it will be published elsewhere. 
to refer to these spaces as "exotic" spaces to distinguish them from the previously known spaces, which are referred to as "classical" spaces. Among other examples, it has been shown that for each $p, 1<p<\infty$, the sequence space $\ell_{p}$ can be obtained as a quotient of a separable, reflexive, hereditarily indecomposable space [ArgyrosF:00]. Many other examples have been constructed. See for example, [ArgyrosLT:03], [ArgyrosT:04], [Koszmider:04], [Koszmider:05] and [Koszmider:05a]. We refer to [Maurey:03] for a readable paper describing many interesting examples of spaces of this kind.

For a complex, infinite dimensional, hereditarily indecomposable Banach space $X$, the quotient algebra $\mathcal{L}(X) / \mathcal{S} \mathcal{S}(X)$ is one-dimensional. Here $\mathcal{S} \mathcal{S}(X)$ is the ideal of strictly singular operators acting on $X$. As a consequence, the essential spectrum of each operator $T \in \mathcal{L}(X)$ consists of just one point. This is quite different from what happens for the classical Banach spaces. So these spaces are a good bench-mark for the development of Fredholm theory, as well as a source of nontrivial examples of operators. The hereditarily indecomposable Banach spaces have also found applications in the study of $C_{0}$-semigroups of operators. See [RabigerR:96] and [RabigerR:98].

In this paper we deal with closed two-sided ideals $\mathcal{A}(X)$ in $\mathcal{L}(X)$ for which an operator $T \in \mathcal{L}(X)$ is Fredholm if and only if the corresponding class is invertible in $\mathcal{L}(X) / \mathcal{A}(X)$. In this case we say that $\mathcal{L}(X) / \mathcal{A}(X)$ is the Calkin algebra associated to the ideal $\mathcal{A}(X)$.

It follows from well-known results that $\mathcal{L}(X) / \mathcal{A}(X)$ is a Calkin algebra if and only if $\mathcal{A}(X)$ contains the finite range operators and is contained in the inessential operators. As examples, we mention the compact, the strictly singular, the strictly cosingular and the inessential operators. Also the perturbation classes in Fredholm theory can be taken as examples.

We are interested in the spaces which admit a Calkin algebra which is "small" in some sense: finite dimensional, commutative, etc. For the reader of this paper, it should become clear that there are plenty of spaces of this kind, even among the $C(K)$ spaces of continuous functions defined on a compact space $K$. In the paper we show the main properties of these spaces, describe some examples and give references for further properties and examples. Our aim is to show that these spaces are useful and not too exotic. So it pays out to have them in mind while working in operator theory.

Let us see a more detailed description of the paper. After a preliminary section, we introduce in Section 3 the concept of Calkin algebra $\mathcal{L}(X) / \mathcal{A}(X)$ associated to some ideal $\mathcal{A}(X)$ of $\mathcal{L}(X)$. We give several examples, show the relations among them and give information on the Calkin algebras of some classical sequence spaces and function spaces.

Section 4 deals with the "decomposability" properties of a Banach space as the direct sum of infinite dimensional closed subspaces. We show the relation of these properties with the number of connected components of the essential spectrum of the operators $T \in \mathcal{L}(X)$. We also show that the spaces whose subspaces (quotients) are decomposable admit characterizations in terms of the classes of operators in Fredholm theory.

Section 5 is dedicated to the Calkin algebra associated to the ideal of inessential operators $\mathcal{L}(X) / \mathcal{I} n(X)$, which is the smallest Calkin algebra. In the case $\mathcal{L}(X) / \mathcal{I} n(X)$ is finite dimensional, we obtain some results on the structure of the space $X$ and describe the essential spectrum of the operators $T \in \mathcal{L}(X)$. Also, in this case we give a 
description of the Calkin algebra $\mathcal{L}(X) / \mathcal{I} n(X)$ as a finite product of algebras of matrices $M_{k}(\mathcal{C})$. This description of $\mathcal{L}(X) / \mathcal{I} n(X)$ allows us to give examples of spaces $X$ for which $\mathcal{L}(X) / \mathcal{S S}(X)$ or $\mathcal{L}(X) / \mathcal{S C}(X)$ can be represented as special subalgebras of $M_{n}(\mathcal{C})$; for example, as triangular algebras.

We have chosen to include all the examples in Section 6. In this way we can appreciate directly the variety of Banach spaces with small Calkin algebras. However, we refer to these examples in the sections in which they are relevant. In order the paper be useful, we give a rough description of some of the constructions, and most of the times we give a reference in which the reader can find more details.

Throughout we refer to other results of the paper by giving the number of the result and the section in which it is stated. For example, Theorem 4.3 is Theorem 3 in section 4.

2. Preliminaries. This paper is a blend of Fredholm theory and Banach space theory. So we have to consider definitions and results in both theories. In this section we include definitions and notations for the classes of operators of Fredholm theory. The corresponding definitions of Banach space theory are introduced when needed.

Let $X$ and $Y$ be Banach spaces. For a subspace $M$ of $X$ we denote by $J_{M}$ the embedding map from $M$ into $X$, and by $Q_{M}$ the quotient map onto $X / M$. We denote by $\mathcal{L}(X, Y)$ the space of continuous operators from $X$ to $Y$, and $\mathcal{K}(X, Y)$ and $\mathcal{F}(X, Y)$ denote the space of compact and finite dimensional range operators, respectively.

Let $T \in \mathcal{L}(X, Y)$.

- $T$ is upper semi-Fredholm, $T \in \Phi_{+}$, if $R(T)$ is closed and $\operatorname{dim} N(T)<\infty$.

- $T$ is lower semi-Fredholm, $T \in \Phi_{-}$, if $R(T)$ is closed and $\operatorname{dim} Y / R(T)<\infty$.

- $T$ is Fredholm, $T \in \Phi$, if $T$ is upper and lower semi-Fredholm.

- For an operator $T \in \mathcal{L}(X)$ the essential spectrum is defined by

$$
\sigma_{e}(T):=\{\lambda \in \mathbb{C}: \lambda I-T \notin \Phi(X)\} .
$$

- The index of a semi-Fredholm operator $T \in \Phi_{+}(X, Y) \cup \Phi_{-}(X, Y)$ is defined by

$$
\operatorname{ind}(T):=\operatorname{dim} N(T)-\operatorname{dim} Y / R(T) .
$$

- $T$ is strictly singular, $T \in \mathcal{S S}$, if given a subspace $M$ of $X, T J_{M}$ isomorphism (into) implies $\operatorname{dim} M<\infty$.

- $T$ is strictly cosingular, $T \in \mathcal{S C}$, if given a subspace $N$ of $Y, Q_{N} T$ surjective implies $\operatorname{dim} Y / N<\infty$.

- $T$ is inessential, $T \in \mathcal{I} n$, if $I-S T \in \Phi(X)$ for all $S \in \mathcal{L}(Y, X)$.

The perturbation class $P \mathcal{C}$ of a class of operators $\mathcal{C}$ was defined by Lebow and Schechter [LebowS:71] as follows. Suppose that $\mathcal{C}(X, Y)$ is a non-empty subset of $\mathcal{L}(X, Y)$. Then

$$
P \mathcal{C}(X, Y):=\{K \in L(X, Y): K+T \in \mathcal{C}(X, Y) \text {, for all } T \in \mathcal{C}(X, Y)\} .
$$

Note that in the case $X=Y, \Phi(X), \Phi_{+}(X)$ and $\Phi_{-}(X)$ are always non-empty.

Proposition 1 ([LebowS:71]). The perturbation classes $P \Phi(X), P \Phi_{+}(X)$ and $P \Phi_{-}(X)$ are closed, two-sided ideals in $\mathcal{L}(X)$. 
3. Calkin algebras. In this section we introduce the concept of Calkin algebra. We also give several examples of Calkin algebras and describe the relations among them.

Definition 1. Let $\mathcal{A}(X)$ be a closed ideal in $\mathcal{L}(X)$. We say that $\mathcal{L}(X) / \mathcal{A}(X)$ is a Calkin algebra whenever the Fredholm operators on $X$ coincide with the operators whose class in $\mathcal{L}(X) / \mathcal{A}(X)$ is invertible.

The following characterization can be easily derived from standard results in Fredholm theory.

Proposition 1. Let $\mathcal{A}(X)$ be a closed ideal in $\mathcal{L}(X)$. Then $\mathcal{L}(X) / \mathcal{A}(X)$ is a Calkin algebra if and only if $\overline{\mathcal{F}(X)} \subset \mathcal{A}(X) \subset \mathcal{I} n(X)$.

The existence of spaces $X$ for which $\overline{\mathcal{F}(X)} \neq \mathcal{K}(X)$ is related with the approximation property of Banach spaces. Since this property has no relevance here, $\mathcal{K}(X)$ is the smallest ideal we consider.

REMARK 1. Roughly, we are going to consider two kinds of closed ideals in $\mathcal{L}(X)$ :

1. Intrinsically defined ideals, for which the fact that the operator belongs to the ideal can be expressed in terms of its action on the spaces in which it is defined, like $\mathcal{K}$, $\mathcal{S S}$ and $\mathcal{S C}$.

2. Perturbation classes in which the definition involves the properties of a large class of operators.

Remark 2. The spaces $\mathcal{K}(X, Y), \mathcal{S S}(X, Y), \mathcal{S C}(X, Y)$ and $\mathcal{I} n(X, Y)$ are defined for all pairs of Banach spaces $X$ and $Y$. They form operator ideals in the sense of [PietschBook:80]. However, the perturbation classes $P \Phi(X, Y), P \Phi_{+}(X, Y)$ and $P \Phi_{-}(X, Y)$ are defined only when $\Phi(X, Y), \Phi_{+}(X, Y)$ and $\Phi_{-}(X, Y)$ are non-empty, respectively.

Next we describe the relations of containment between the main ideals that we consider in the paper.

Proposition 2. The following results hold whenever the perturbation classes are defined.

- $\mathcal{I} n(X, Y)=P \Phi(X, Y) \quad[$ Kleinecke:63].

- $P \Phi_{+}(X, Y) \cup P \Phi_{-}(X, Y) \subset P \Phi(X, Y)$ (Stability of the index).

- $\mathcal{S S}(X, Y) \subset P \Phi_{+}(X, Y) \quad[$ Kato:58].

- $\mathcal{S C}(X, Y) \subset P \Phi_{-}(X, Y) \quad$ [Vladimirskii:67].

The following diagram shows the relations between the ideals we have introduced before.

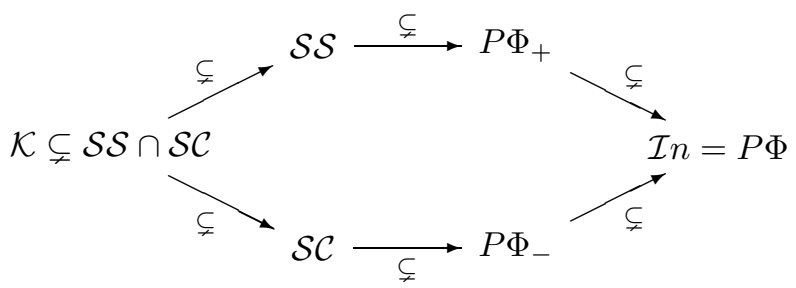


There are natural operators that show that all the inclusions, except $\mathcal{S S} \subsetneq P \Phi_{+}$and $\mathcal{S C} \subsetneq P \Phi_{-}$, are proper. Indeed, let us consider the embedding maps

$$
L_{\infty}[0,1] \stackrel{J_{2}}{\longrightarrow} L_{2}[0,1] \stackrel{J_{1}}{\longrightarrow} L_{1}[0,1] .
$$

Then $J_{1} J_{2} \in(\mathcal{S S} \cap \mathcal{S C}) \backslash \mathcal{K}, J_{2} \in(\mathcal{S S} \backslash \mathcal{S C}), J_{1} \in(\mathcal{S C} \backslash \mathcal{S S})$ and $J_{1}, J_{2} \in \mathcal{I} n$. We refer to Proposition 2.6 in [Gonzalez:04] for details.

For the remaining inclusions we need exotic spaces. It was shown in [Gonzalez:03] that there exists a reflexive Banach space $X$, which can be defined in terms of products of subspaces of $X_{G M}$, such that $\mathcal{S S}(X) \neq P \Phi_{+}(X)$ and $\mathcal{S C}\left(X^{*}\right) \neq P \Phi_{-}\left(X^{*}\right)$. See [Gonzalez:03] for details.

REMARK 3. It follows from the inequalities $\mathcal{S S}(X) \neq P \Phi_{+}(X)$ and $\mathcal{S C}\left(X^{*}\right) \neq P \Phi_{-}\left(X^{*}\right)$ that the perturbation classes $P \Phi_{+}(X, Y)$ and $P \Phi_{-}(X, Y)$ for operators acting between different spaces do not give operators ideals. See the Remark in section 26.6 of [PietschBook:80].

REMARK 4. It is not known if there exists an infinite dimensional Banach space $X$ for which $\operatorname{dim} \mathcal{L}(X) / \mathcal{K}(X)<\infty$. This is a (probably hard) open problem in Banach space theory.

REMARK 5. The definition of the inessential operators is not intrinsic. In order to check if $T \in \mathcal{I} n(X, Y)$, we have to consider the properties of the product $S T$ of $T$ with all the operators $S \in \mathcal{L}(Y, X)$.

Trying to obtain an intrinsic characterization of the inessential operators, Tarafdar introduced in [Tarafdar:72] the improjective operators. An operator $T \in \mathcal{L}(X, Y)$ is said to be improjective, $T \in \mathcal{I} m p(X, Y)$, if there is no infinite dimensional subspace $M$ of $X$ such that the restriction $T J_{M}$ is an isomorphism and $T(M)$ is complemented in $Y$. It is not difficult to show that $\mathcal{I} n(X, Y) \subset \mathcal{I} m p(X, Y)$.

The equality $\mathcal{I} n(X, Y)=\mathcal{I} m p(X, Y)$ holds for many pairs of spaces (see [AienaG:98]). However, this inclusion is proper in general: an improjective not inessential operator was given in [AienaG:00]. The construction is based on the properties of the shift operator acting in the space described below as Example 6.1.2.

REMARK 6. For classical Banach spaces the Calkin algebras are big spaces, in general. However we can give some interesting facts.

- For $X$ one of the sequence spaces $c_{0}$ and $\ell_{p}, 1 \leq p<\infty$, there is only one Calkin algebra. Equivalently, $\overline{\mathcal{F}(X)}=\mathcal{K}(X)=\mathcal{I} n(X)$. Moreover, $\mathcal{L}(X) / \mathcal{K}(X)$ is nonseparable. In [CaradusPY:74] we can find a proof of this result and some additional details.

- For $X$ a subprojective space, like the function spaces $L_{p}(0,1) ; 1 \leq p<2, \mathcal{S S}(X)=$ $\mathcal{I} n(X)$; and for $X$ a superprojective space, like the function spaces $L_{p}(0,1) ; 2 \leq$ $p<\infty, \mathcal{S C}(X)=\mathcal{I} n(X)$. See [CaradusPY:74] and [Weis:81].

4. Finitely decomposable spaces. In this section we study the relation between the decomposability of a Banach space $X$ as a direct sum of closed infinite dimensional subspaces and the properties of the Calkin algebras $\mathcal{L}(X) / \mathcal{A}(X)$. 
Definition 1. Let $X$ be a Banach space.

- $X$ is indecomposable if it cannot be written as the direct sum of two closed infinite dimensional subspaces.

- $X$ is $n$-decomposable if $n=\max \left\{k \in \mathbb{N}: X=X_{1} \oplus \cdots \oplus X_{k}\right\}$, where $X_{1}, \ldots, X_{n}$ are closed infinite dimensional subspaces of $X$.

- $X$ is hereditarily indecomposable if all its closed subspaces are indecomposable.

- $X$ is quotient indecomposable if all its quotients are indecomposable.

As an application of the analytic functional calculus for operators (see for example [TaylorL:80]) we can prove the following characterizations.

Theorem 1 ([GonzalezH:03]). Let $X$ be a complex Banach space. Then $X$ is indecomposable if and only if $\sigma_{e}(T)$ is connected for all $T \in \mathcal{L}(X)$. Similarly, $X$ is n-decomposable if and only if $n$ is the maximum of the number of connected components of $\sigma_{e}(T)$ for $T \in \mathcal{L}(X)$.

The first part of the following characterizations was proved in [Weis:81] in 1981. Observe that at that time no example of hereditarily indecomposable or quotient indecomposable space was known.

TheOREm 2 (see [Ferenczi:97]). A Banach space $X$ is hereditarily indecomposable if and only if, for every Banach space $Y$,

$$
\mathcal{L}(X, Y)=\Phi_{+}(X, Y) \cup \mathcal{S S}(X, Y) .
$$

In the complex case, this is equivalent to the fact that for each closed subspace $M$ of $X$ and every $T \in \mathcal{L}(M, X)$ there exists $\lambda \in \mathcal{C}$ such that $T-\lambda J_{M}$ is strictly singular.

Theorem 3 (see [GonzalezH:01]). A Banach space $X$ is quotient indecomposable if and only if, for every Banach space $Z$,

$$
\mathcal{L}(Z, X)=\Phi_{-}(Z, X) \cup \mathcal{S C}(Z, X) .
$$

In the complex case, this is equivalent to the fact that for each quotient $X / N$ of $X$ and every $T \in \mathcal{L}(X, X / N)$ there exists $\lambda \in \mathcal{C}$ such that $T-\lambda Q_{N}$ is strictly cosingular.

REMARK 1. The classical Banach spaces are far from being indecomposable. However, many examples of indecomposable spaces have been found by now.

- The space $X_{G M}$, which appears here as Example 6.1.1, is hereditarily indecomposable and quotient indecomposable. See [Ferenczi:99].

- The space $X_{s}$ of Example 6.1.2 is indecomposable, but not hereditarily indecomposable or quotient indecomposable.

- Koszmider's space in Example 6.2 is an indecomposable $C(K)$ space, but contains subspaces isomorphic to $c_{0}$ and admits quotients isomorphic to $\ell_{2}$.

It has been open for some time whether a hereditarily indecomposable space has to be separable. Finally, a negative answer has been obtained. However, some limitations apply. 
THEOREM 4 ([ArgyrosT:04]). There are nonseparable hereditarily indecomposable Banach space; however, each hereditarily indecomposable Banach space is isomorphic to a subspace of $\ell_{\infty}$.

5. The "smallest" Calkin algebra. Here we deal with $\mathcal{L}(X) / \mathcal{I} n(X)$, which is the smallest Calkin algebra. Throughout this section, $X$ is a complex Banach space.

REMARK 1. The inessential operators were introduced in [Kleinecke:63] as follows. Let $\pi: \mathcal{L}(X) \rightarrow \mathcal{L}(X) / \mathcal{K}(X)$ denote the quotient map. Then $\mathcal{I} n(X)$ is the inverse under $\pi$ of the radical of $\mathcal{L}(X) / \mathcal{K}(X)$.

The following classes of operators were introduced in [Atkinson:51].

Definition 1. Let $T \in \mathcal{L}(X, Y)$.

- $T$ is left Atkinson, denoted $T \in \Phi_{l}$, if $R(T)$ is complemented and $\operatorname{dim} N(T)<\infty$.

- $T$ is right Atkinson, denoted $T \in \Phi_{r}$, if $N(T)$ is complemented $R(T)$ closed and $\operatorname{dim} Y / R(T)<\infty$.

REMARK 2. The relevance of the Atkinson operators in this section stems from the following facts. Let $\mathcal{L}(X) / \mathcal{A}(X)$ be a Calkin algebra.

1. $T \in \mathcal{L}(X)$ is left Atkinson if and only if the class of $T$ in $\mathcal{L}(X) / \mathcal{A}(X)$ is left invertible.

2. $T \in \mathcal{L}(X)$ is right Atkinson if and only if the class of $T$ in $\mathcal{L}(X) / \mathcal{A}(X)$ is right invertible.

3. $P \Phi_{l}(X)=P \Phi_{r}(X)=P \Phi(X)=\mathcal{I} n(X)$.

We refer to [LebowS:71] or [CaradusPY:74] for proofs of these facts.

Let us see how the size of $\mathcal{L}(X) / \mathcal{I} n(X)$ is directly related to the size of the essential spectrum of the operators acting on $X$.

Let $|A|$ denote the cardinal of a set $A$. We introduce the class $\Sigma_{e}^{n}$ as follows.

$$
\Sigma_{e}^{n}:=\left\{X: n=\max \left\{\left|\sigma_{e}(T)\right|: T \in \mathcal{L}(X)\right\}\right\} .
$$

TheOREM 1 ([GonzalezH:05]). The following assertions are equivalent:

1. For every $T \in \mathcal{L}(X)$, the essential spectrum $\sigma_{e}(T)$ is finite.

2. $\operatorname{dim} \mathcal{L}(X) / \mathcal{I} n(X)<\infty$.

3. The space $X$ belongs to the class $\Sigma_{e}^{n}$, for some $n \in \mathbb{N}$.

REMARK 3. It is not difficult to see that $X$ belongs to the class $\Sigma_{e}^{1}$ if and only if $\operatorname{dim} \mathcal{L}(X) / \mathcal{I} n(X)=1$.

Definition 1. Let $X$ and $Y$ be Banach spaces.

- We say that $X$ and $Y$ are essentially incomparable when $\mathcal{L}(X, Y)=\mathcal{I} n(X, Y)$.

- We say that $X$ and $Y$ are essentially isomorphic when $\Phi(X, Y)$ is non-empty.

REMARK 4. The concept of essential incomparability is "symmetric" [Gonzalez:94]; i.e., $\mathcal{L}(X, Y)=\mathcal{I} n(X, Y)$ if and only if $\mathcal{L}(Y, X)=\mathcal{I} n(Y, X)$.

Remark 5. $X$ essentially isomorphic to $Y$ means that $X$ is isomorphic to $Y$ up to a finite dimensional space. 
There are many examples of pairs of Banach spaces which are essentially incomparable.

TheOrem 2 ([Gonzalez:94]). In the following cases, the spaces $X$ and $Y$ are essentially incomparable.

1. $X$ is reflexive and $Y$ has the Dunford-Pettis property.

2. $X$ has the reciprocal Dunford-Pettis property and $Y$ has the Schur property.

3. $X$ contains no copies of $\ell_{\infty}$ and $Y=\ell_{\infty}$ or $C(K)$ with $K \sigma$-stonian.

4. $X$ contains no copies of $c_{0}$ and $Y=C(K)$.

5. $X$ contains no complemented copies of $c_{0}$ and $Y=C[0,1]$.

6. $X$ contains no complemented copies of $\ell_{1}$ and $Y=L_{1}[0,1]$.

7. $X$ contains no complemented copies of $\ell_{p}$ and $Y=L_{p}[0,1]$, or $\ell_{p} ; 1<p<\infty$.

ExAmple 1. Let $X$ be a hereditarily indecomposable space and let $M$ be a subspace of $X$ such that $\operatorname{dim} X / M=\infty$. It follows from Theorem 4.2 that $M$ and $X$ are essentially incomparable.

Similarly, let $X$ be a quotient indecomposable space and let $M$ be an infinite dimensional subspace of $X$. It follows from Theorem 4.3 that $X$ and $X / M$ are essentially incomparable.

The following result should be compared with the corresponding results for hereditarily indecomposable spaces (Theorem 4.2) and quotient indecomposable spaces (Theorem 4.3).

Theorem 3 ([GonzalezH:05]). For an infinite dimensional Banach space $X$, the following assertions are equivalent.

1. $\operatorname{dim} \mathcal{L}(X) / \mathcal{I} n(X)=1$; i.e., $X \in \Sigma_{e}^{1}$.

2. $\mathcal{L}(X)=\Phi(X) \cup \mathcal{I} n(X)$.

3. $\mathcal{L}(X, Y)=\Phi_{l}(X, Y) \cup \mathcal{I} n(X, Y)$, for all $Y$.

4. $\mathcal{L}(Z, X)=\Phi_{l}(Z, X) \cup \mathcal{I} n(Z, X)$, for all $Z$.

Next we give a result that provides a precise description of the spaces $X$ for which $\mathcal{L}(X) / \mathcal{I} n(X)$ is finite dimensional.

Theorem 4 ([GonzalezH:05], Structure theorem). Suppose that $X \in \Sigma_{e}^{n}$. Then there exist $k$ integers $n_{i}$ and $k$ spaces $X_{1}, \ldots, X_{k}$ satisfying the following properties.

- $n=n_{1}+\cdots+n_{k}$.

- $X_{i}$ and $X_{j}$ are essentially incomparable for $i \neq j$.

- $X$ is essentially isomorphic to $\left(n_{1}\right.$ copies of $\left.X_{1}\right) \oplus \cdots \oplus\left(n_{k}\right.$ copies of $\left.X_{k}\right)$.

As a consequence of Theorem 4 we obtain a description of $\mathcal{L}(X) / \mathcal{I} n(X)$.

Theorem 5 ([GonzalezH:05]). Suppose that $X \in \Sigma_{e}^{n}$. Then there exist $k$ integers $n_{1}, \ldots, n_{k}$ satisfying $n=n_{1}+\cdots+n_{k}$ so that

$$
\mathcal{L}(X) / \mathcal{I} n(X) \text { is isomorphic to } M_{n_{1}}(\mathcal{C}) \oplus \cdots \oplus M_{n_{k}}(\mathcal{C}) .
$$

Theorems 4 and 5 allow us to give examples of spaces with different Calkin algebras. 
EXAmPLE 2. Let us consider the space $X_{G M}$ in Example 6.1.1. Since $X_{G M}$ is hereditarily indecomposable, taking infinite dimensional closed subspaces $M_{1} \subset \cdots \subset M_{n}$ of $X_{G M}$ such that $\operatorname{dim} M_{k+1} / M_{k}=\infty$ for $k=1, \ldots, n-1$ and denoting

$$
Y:=M_{1} \oplus \cdots \oplus M_{n},
$$

then $\mathcal{L}(Y) / \mathcal{I} n(Y)$ can be identified with the diagonal $n \times n$ complex matrices, while $\mathcal{L}(Y) / \mathcal{S S}(Y)$ can be identified with the triangular $n \times n$ complex matrices.

Similarly, since $X_{G M}$ is quotient indecomposable, taking closed subspaces $M_{1} \supset \cdots \supset$ $M_{n+1}$ of $X_{G M}$ such that $\operatorname{dim} M_{k} / M_{k+1}=\infty$ for $k=1, \ldots, n$, and denoting

$$
Z:=M_{1} / M_{2} \oplus \cdots \oplus M_{n} / M_{n+1}
$$

then $\mathcal{L}(Z) / \mathcal{I} n(Z)$ can be identified with the diagonal $n \times n$ complex matrices, while $\mathcal{L}(Z) / \mathcal{S C}(Z)$ can be identified with the triangular $n \times n$ complex matrices.

6. The examples. In this section we present some examples of exotic Banach spaces that are relevant as examples or counterexamples in Fredholm theory.

As usual, we denote by $\mathbb{D}$ and $\mathbb{T}$ the unit disc and the unit circle in the complex field, respectively.

\subsection{The Gowers-Maurey examples [Gowers-Maurey:97]}

1. (The HI and QI space) There exists a reflexive, complex hereditarily indecomposable Banach space $X_{G M}$ such that

$$
\operatorname{dim} \mathcal{L}\left(X_{G M}\right) / \mathcal{S S}\left(X_{G M}\right)=\operatorname{dim} \mathcal{L}\left(X_{G M}^{*}\right) / \mathcal{S C}\left(X_{G M}^{*}\right)=1 .
$$

Even more, for every closed subspace $Y$ of $X_{G M}$ and every $T \in \mathcal{L}\left(Y, X_{G M}\right)$, there exists a complex number $\lambda$ such that $T-\lambda J_{Y} \in \mathcal{S S}\left(Y, X_{G M}\right)$. It was shown in [Ferenczi:99] that the space $X_{G M}$ is also quotient indecomposable. Thus

$$
\operatorname{dim} \mathcal{L}\left(X_{G M}\right) / \mathcal{S C}\left(X_{G M}\right)=\operatorname{dim} \mathcal{L}\left(X_{G M}^{*}\right) / \mathcal{S S}\left(X_{G M}^{*}\right)=1 .
$$

Moreover, for every quotient $Z$ of $X_{G M}$ and every $T \in \mathcal{L}\left(X_{G M}, Z\right)$, there exists a complex number $\lambda$ such that $T-\lambda Q \in \mathcal{S C}\left(X_{G M}, Z\right)$, where $Q$ is the quotient map onto $Z$.

2. (The shift space) There exists a complex Banach space $X_{s}$ which admits a Schauder basis and satisfies the following properties:

(a) $\mathcal{L}\left(X_{s}\right)$ admits a closed ideal $\mathcal{A}\left(X_{s}\right)$ with $\mathcal{K}\left(X_{s}\right) \subset \mathcal{A}\left(X_{s}\right) \subset \mathcal{S S}\left(X_{s}\right)$.

(b) Denoting by $S$ and $L$ the right shift and the left shift acting on $X_{s}$,

$$
\sigma(S)=\sigma(L)=\mathbb{D} \quad \text { and } \quad \sigma_{e}(S)=\sigma_{e}(L)=\mathbb{T} .
$$

(c) For every operator $T \in \mathcal{L}\left(X_{s}\right)$, there exists a sequence $\left(a_{n}\right)_{n=-\infty}^{\infty}$ of complex numbers such that $\sum_{n=-\infty}^{\infty}\left|a_{n}\right|<\infty$ and

$$
T-\sum_{n=0}^{\infty} a_{n} S^{n}-\sum_{n=1}^{\infty} a_{-n} L^{n} \in \mathcal{S S}\left(X_{s}\right) .
$$

(d) Denoting by $\Psi_{T}$ the function given by $\Psi_{T}(\lambda):=\sum_{n=-\infty}^{\infty} a_{n} \lambda^{n}$,

$$
\sigma(T)=\Psi_{T}(\mathbb{D}) \quad \text { and } \quad \sigma_{e}(T)=\Psi_{T}(\mathbb{T}) .
$$


(e) $\mathcal{L}\left(X_{s}\right) / \mathcal{A}\left(X_{s}\right)$ can be identified with the algebra $\ell_{1}(\mathbb{Z})$, endowed with the convolution product. Indeed, if $T=\sum_{n=0}^{\infty} a_{n} S^{n}-\sum_{n=1}^{\infty} a_{-n} L^{n}$ is strictly singular, then $\sigma_{e}(T)=\Psi_{T}(\mathbb{T})=\{0\}$. Thus $T=0$.

(f) Each pair of operators $A, B \in \mathcal{L}\left(X_{s}\right)$ commute modulo the strictly singular operators; i.e., $A B-B A \in \mathcal{S S}\left(X_{s}\right)$.

3. (The double shift space) There exists a complex Banach space $X_{d s}$ which is isomorphic to its closed subspaces of even codimension while not being isomorphic to those of odd codimension. As a consequence, each Fredholm operator acting on $X_{d s}$ has even index.

4. There exists a Banach space $X$ which is isomorphic to $X \oplus X \oplus X$ but is not isomorphic to $X \oplus X$.

5. There exists a Banach space $X_{d}$ which has an unconditional Schauder basis and satisfies the following properties.

- Every operator on $X_{d}$ is the sum of a diagonal operator and a strictly singular one.

- $\mathcal{L}\left(X_{d}\right) / \mathcal{S S}\left(X_{d}\right)$ can be identified with $\ell_{\infty} / c_{0}$. See [Ferenczi:95].

REMARK 1. The constructions of the previous Examples 1-5 admit modifications and extensions to obtain other Banach spaces satisfying nonclassical properties. We refer to the paper [GowersM:97] for further details.

6.2. The Koszmider example [Koszmider:04]. There exists a connected, separable compact space $K$ such that the space $C(K)$ of real-valued continuous defined on $K$ satisfies the following properties:

(a) $C(K)$ is indecomposable and is not isomorphic to any of its proper subspaces or quotients.

(b) It follows from property (a) that every semi-Fredholm operator on $C(K)$ is Fredholm with index zero.

(c) For every operator $T \in \mathcal{L}(C(K))$ there exists a function $\phi \in C(K)$ such that $T=M_{\phi}+S$, where $M_{\phi}$ is the multiplication operator by $\phi$ and $S$ is a strictly singular operator.

(d) It follows from property (c) that the Calkin algebra $\mathcal{L}(C(K)) / \mathcal{S} \mathcal{S}(C(K))$ can be identified with the space $C(K)$. Indeed, since $K$ is connected, if $M_{\phi}$ is strictly singular then $\phi(K)=\{0\}$, hence $M_{\phi}=0$.

REMARK 2. Koszmider has found some other examples of $C(K)$ spaces whose properties are remarkable from the point of view we consider here. See [Koszmider:05] and [Koszmider:05a].

6.3. The Argyros et al. examples. In the last few years, S. Argyros and coworkers have found plenty of examples of hereditarily indecomposable and quotient indecomposable Banach spaces.

1. There exists a reflexive, separable, hereditarily indecomposable Banach space $X$ which admits a quotient isomorphic to $\ell_{2}$ [ArgyrosF:00]. Hence, the dual space $X^{*}$ is quotient indecomposable and contains a subspace isomorphic to $\ell_{2}$. 
2. There exists a Banach space $X$ such that $X, X^{*}$ and $X^{* *}$ are hereditarily indecomposable, $X$ and $X^{*}$ are separable but $X^{* *}$ is not separable [ArgyrosT:04].

The quotient space $X^{* *} / X$ is isomorphic to $c_{0}(\Gamma)$, where $\Gamma$ is an uncountable set. Every operator $T \in \mathcal{L}(X)$ can be written as $T=\lambda I+S$, where $\lambda$ is a real number and $S$ is strictly cosingular and weakly compact.

Every operator $T \in \mathcal{L}\left(X^{*}\right)$ can be written as $T=\lambda I+S$, where $\lambda$ is a real number and $S$ is strictly singular and weakly compact.

Every operator $T \in \mathcal{L}\left(X^{* *}\right)$ can be written as $T=S^{*}+K$, where $S \in \mathcal{L}\left(X^{*}\right)$ and $K$ is compact.

We refer to [ArgyrosDKM:98], [ArgyrosLT:03], [ArgyrosM:03] and the above mentioned papers for further examples and additional information.

Acknowledgements. The author thanks J. M. Herrera and the referee for their helpful comments.

\section{References}

[AienaG:98] P. Aiena and M. González, On inessential and improjective operators, Studia Math. 131 (1998), 271-287.

[AienaG:00] P. Aiena and M. González, Examples of improjective operators, Math. Z. 233 (2000), 471-479.

[ArgyrosDKM:98] S. A. Argyros, I. Deliyanni, D. Kutzarova and A. Manoussakis, Modified mixed Tsirelson spaces, J. Funct. Anal. 159 (1998), 43-109.

[ArgyrosF:00] S. A. Argyros and V. Felouzis, Interpolating hereditarily indecomposable Banach spaces, J. Amer. Math. Soc. 13 (2000), 243-294.

[ArgyrosLT:03] S. A. Argyros, J. Lopez-Abad and S. Todorcevic, A class of Banach spaces with no unconditional basic sequence, C. R. Math. Acad. Sci. Paris 337 (2003), 43-48.

[ArgyrosM:03] S. A. Argyros and A. Manoussakis, An indecomposable and unconditionally saturated Banach space, Studia Math. 159 (2003), 1-32.

[ArgyrosT:04] S. A. Argyros and A. Tolias, Methods in the theory of hereditarily indecomposable Banach spaces, Mem. Amer. Math. Soc. 170 (2004), no. 806.

[Atkinson:51] F. Atkinson, The normal solvability of linear equations in normed spaces, Mat. Sbornik 28 (1951), 3-14 (in Russian).

[CaradusPY:74] S. Caradus, W. Pfaffenberger and B. Yood, Calkin Algebras and Algebras of Operators in Banach Spaces, Lecture Notes in Pure \& Appl. Math. 9, M. Dekker, New York, 1974.

[Ferenczi:95] V. Ferenczi, Quelques propriétés des espaces de Banach héréditairement indécomposables, Ph. D. Thesis, Université de Paris 1, 1995.

[Ferenczi:97] V. Ferenczi, Operators on subspaces of hereditarily indecomposable Banach spaces, Bull. London Math. Soc. 29 (1997), 338-344.

[Ferenczi:99] V. Ferenczi, Quotient hereditarily indecomposable Banach spaces. Canad. J. Math. 51 (1999), 566-584.

[Gonzalez:94] M. González, On essentially incomparable Banach spaces, Math. Z. 215 (1994), 621-629. 
[Gonzalez:03]

[Gonzalez:04]

[GonzalezH:01]

[GonzalezH:03]

[GonzalezH:05]

[GowersM:93]

[GowersM:97]

[Kato:58]

[Kleinecke:63]

[Koszmider:04]

[Koszmider:05]

[Koszmider:05a]

[LebowS:71]

[Maurey:03]

[PietschBook:80]

[RabigerR:96]

[RabigerR:98]

[Tarafdar:72]

[TaylorL:80]

[Vladimirskii:67]

[Weis:81]
M. González, The perturbation classes problem in Fredholm theory, J. Funct. Anal. 200 (2003), 65-70.

M. González, The perturbation classes of some semigroups associated to an operator ideal, Rendiconti Circ. Mat. Palermo, Ser. II 73 (2004), 53-75.

M. González and J. M. Herrera, Operators on quotient indecomposable spaces, Publ. Math. Debrecen 59 (2001), 271-288.

M. González and J. M. Herrera, Calkin algebras for Banach spaces with finitely decomposable quotients, Studia Math. 157 (2003), 279-293.

M. González and J. M. Herrera, Spaces on which all the operators have finite essential spectrum, J. Operator Theory 53 (2005), 303-314.

W. T. Gowers and B. Maurey, The unconditional basic sequence problem, J. Amer. Math. Soc. 6 (1993), 851-874.

W. T. Gowers and B. Maurey, Banach spaces with small spaces of operators, Math. Ann. 307 (1997), 543-568.

T. Kato, Perturbation theory for nullity, deficiency and other quantities of linear operators, J. d'Analyse Math. 6 (1958), 261-322.

D. Kleinecke, Almost finite, compact and inessential operators, Proc. Amer. Math. Soc. 14 (1963), 863-868.

P. Koszmider, Banach spaces of continuous functions with few operators, Math. Ann. 330 (2004), 151-183.

P. Koszmider, A space $C(K)$ where all non-trivial complemented subspaces have big densities. Studia Math. 168 (2005), 109-127.

P. Koszmider, On decompositions of Banach spaces of continuous functions on Mrówka's spaces. Proc. Amer. Math. Soc. 133 (2005), 2137-2146.

A. Lebow and M. Schechter, Semigroups of operators and measures of noncompactness, J. Funct. Anal. 7 (1971), 1-26.

B. Maurey, Banach spaces with few operators, in: Handbook of the Geometry of Banach Spaces, North-Holland, 2003, 1247-1297.

A. Pietsch, Operator Ideals, North-Holland, Amsterdam, 1980.

F. Räbiger and W. J. Ricker, $C_{0}$-groups and $C_{0}$-semigroups of linear operators on hereditarily indecomposable Banach spaces, Arch. Math. 66 (1996), 60-70.

F. Räbiger and W. J. Ricker, $C_{0}$-semigroups and cosine families of linear operators in hereditarily indecomposable Banach spaces, Acta Sci. Math. (Szeged) 64 (1998), 697-706.

E. Tarafdar, Improjective operators and ideals in a category of Banach spaces, J. Austral. Math. Soc. 14 (1972), 274-292.

A. E. Taylor and D. C. Lay, Introduction to Functional Analysis, Wiley, New York, 1980.

J. I. Vladimirskii, Strictly cosingular operators, Soviet Math. Doklady 8 (1967), 739-740.

L. Weis, Perturbation classes of semi-Fredholm operators, Math. Z. 178 (1981), 429-442. 